\title{
Improving Energy Efficiency in Dual-hop Cooperative PLC Relaying Systems
}

\author{
Khaled M. Rabie*, Bamidele Adebisi* and Abdelhamid Salem ${ }^{\ddagger}$ \\ ${ }^{*}$ School of Engineering, Manchester Metropolitan University, Manchester, UK \\ ${ }^{\ddagger}$ School of Electrical \& Electronic Engineering, University of Manchester, Manchester, UK \\ Email: \{k.rabie@mmu.ac.uk; b.adebisi@mmu.ac.uk; abdelhamid.salem@manchester.ac.uk\}
}

\begin{abstract}
Energy efficiency (EE) in multi-hop cooperative communication systems, both wireless and wired, is increasingly becoming more and more critical. This has recently been extended to include power line communications (PLC). In this respect, we propose in this paper to enhance the $\mathrm{EE}$ of a dual-hop amplify-and-forward (AF) cooperative relaying PLC system by considering energy-harvesting $(\mathbf{E H})$ at the relay node. The energy harvester exploits the high noisy PLC channel feature as well as the transmitted signal power to forward the source information. In light of this, we derive an analytical expression for the EE and verify it with Monte Carlo simulations. The performance of the conventional relaying system, i.e. without any $\mathbf{E H}$, is also considered to clearly quantify the achievable gains. The results show that the proposed system can considerably improve the $\mathbf{E E}$ of PLC systems and that increasing the channel variance will always make the proposed system more energy-efficient.
\end{abstract}

Index Terms-AF relaying, cooperative communications, energy efficiency (EE), energy-harvesting (EH), power line communications (PLC).

\section{INTRODUCTION}

$\mathbf{P}$ OWER line communication (PLC) technology has enabled many smart grid applications as well as had the potential to offer high-speed home-networking capabilities. However, the fact that this massive infrastructure was not designed to carry communication signals at high frequencies makes it a hostile communication medium suffering from several impairments such as high levels of non-Gaussian interference, impedance mismatching, multipath fading as well as high frequency-dependent attenuation [1]-[4]. What makes this even worse is the low transmit power restrictions that should comply with the regulations determined by independent and governmental regulatory agencies [5]. As a result, the signal-to-noise ratio (SNR) at the receiving PLC modems can be very low which may significantly degrade communication performance of such systems.

To overcome this problem, different techniques have been reported in the literature, amongst which is cooperative relaying techniques. Although many relaying protocols have been studied in the context of PLC such as amplify-andforward (AF) and decode-and-forward (DF), the former has received more attention mainly due to its simplicity since it simply amplifies the received signals from the source, and forwards them to the destination [6]-[8]. More specifically, the authors in [8] have evaluated the performance of a dualhop AF PLC system and concluded that considerable capacity improvements can be attained in comparison to the direct-link scenario. In addition, [7] studied opportunistic DF in in-home PLC networks where similar conclusions are drawn.

Very recently, however, power consumption in PLC systems, which was found to increase almost linearly with bit-rate [9], has attracted a large amount of research attention [10], [11]. For instance, in [12] and [13], the authors have investigated power consumption in opportunistic DF-based PLC networks and presented that several $\mathrm{dBs}$ of transmitted power can be saved in comparison to the direct-transmission scheme. Also, and unlike the previous studies that studied minimizing only the PLC modem transmit power (dynamic power) while ignoring the static power (the power consumed by PLC modems when no data is being transmitted), the authors in [14] took into consideration both the static power and dynamic power when evaluating the energy efficiency $(\mathrm{EE})^{1}$ performance of a half-duplex DF PLC relaying system.

In contrast to the studies above, in this paper we propose to enhance the EE of in-door PLC systems not by optimizing system parameters to reduce transmit power but by exploiting (scavenging) ${ }^{2}$ the high unwanted energy of the various noise components present over PLC channels as well as the source signal power. Such available energy can be intelligently harvested by relay nodes and then used to forward information. This will, consequently, make the PLC relaying nodes less dependent on external power supplies and hence more energyefficient PLC systems will be obtained. Generally, there are three main energy harvesting $(\mathrm{EH})$ protocols, namely, time switching relaying, power splitting relaying and ideal relaying. Since the latter protocol can process the information signal while harvesting energy concurrently, it will therefore be adopted in this study. The performance of the proposed system is evaluated in terms of the EE for which an accurate analytical expression is derived and validated with Monte Carlo simulations. It should be mentioned that the PLC channel in our analysis is considered to have log-normal distribution as reported in [2] and to characterize the background and impulsive noise, we adopt the well-known Bernoulli-Gaussian noise model [15]. Results have shown that the proposed EHbased relaying PLC system is able to always outperform the conventional relaying approach. In addition, it will be shown that the system will become more energy-efficient as the

\footnotetext{
${ }^{1} \mathrm{EE}$ is defined as the quantity of information transmitted from a transmitter to a receiver per unit energy use.

${ }^{2}$ This is also commonly referred to as energy harvesting (EH).
} 


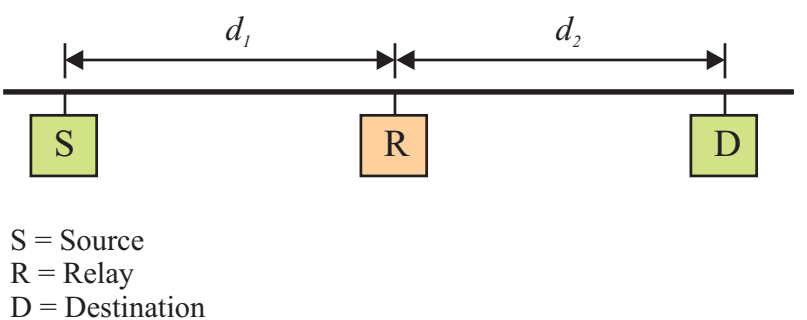

Figure 1: Basic system model with cooperative AF relaying over the PLC channel.

channel variance is increased.

The rest of this paper is organized as follows. Section II presents the system model including the channel model used, noise model, as well as the relaying and EH protocols. In Section III, we analyze the EE of the proposed system. Numerical examples and simulation results to evaluate the system performance are presented and discussed in Section IV. Finally, Section V concludes the paper.

\section{SYSTEM MODEL}

Fig. 1 illustrates the system model under consideration. This system consists of a source node, relaying node and a destination node. The relay is equipped with an energy harvester to harvest the high noise energy over the PLC channel and use it to forward the source information. Below we discuss the channel and noise models deployed in this paper.

\section{A. Channels Model}

The source-to-relay and relay-to-destination channel gains are denoted as $h_{1}$ and $h_{2}$, and the corresponding distances are $d_{1}$ and $d_{2}$, respectively. The two links experience quasistatic block fading; that is channels remain constant over the block time, $T$, and vary independently and identically from one block to another according to log-normal distribution with the following probability density function (PDF)

$$
f_{Z}\left(z_{i}\right)=\frac{\zeta}{\sqrt{2 \pi} \sigma z_{i}} \exp \left[-\frac{\left(10 \log _{10}\left(z_{i}\right)-\mu\right)^{2}}{2 \sigma^{2}}\right]
$$

where $z_{i}=h_{i}^{2}, i \epsilon\{1,2\}, \zeta=10 / \ln (10)$ is a scaling constant, $\mu$ and $\sigma^{2}$ (both in decibels) are the mean and the variance of $10 \log _{10}(h)$, respectively. The moment-generating function (MGF) of this PDF, which will be used later to analyze the system's EE, is calculated as [16, eq. (2.54)]

$$
\Psi_{Z}(s)=\int_{0}^{\infty} \exp (-s z) f(z) \mathrm{d} z
$$

which can also be expanded to

$$
\Psi_{Z}(s)=\int_{0}^{\infty} \frac{\exp (-s z) \zeta}{\sqrt{2 \pi} \sigma z} \exp \left[-\frac{\left(10 \log _{10}(z)-\mu\right)^{2}}{2 \sigma^{2}}\right] \mathrm{d} z
$$

This MGF has no closed-form expression available in the literature. However, it can be expressed, for real $s$, by a series expansion based on Gauss-Hermite integration as follows [17]

$$
\Psi(s) \triangleq \sum_{n=1}^{N} \frac{w_{n}}{\sqrt{\pi}} \exp \left[-s \exp \left(\frac{\sqrt{2} \sigma x_{n}+\mu}{\zeta}\right)\right],
$$

where $N$ denotes the Hermite integration order, $\left\{w_{n}\right\}_{n=1}^{N}$ and $\left\{x_{n}\right\}_{n=1}^{N}$ are the weights and abscissas, respectively. It should be mentioned that in this paper we will use $N=25$, as this was found sufficient to accurately approximate the MGF, and the corresponding weights and abscissas are tabulated in [18, Table 25.10]. Furthermore, to characterize the PLC frequency-dependent attenuation and losses induced by the cable's imperfections, we adopt a simple path loss model given by $\exp (-\alpha d)$, where $\alpha=a_{o}+a_{1} f^{k}$ is the attenuation factor, $a_{o}$ and $a_{1}$ are constants determined from measurements, $f$ is the frequency and $k$ denotes the exponent of the attenuation factor [1].

\section{B. Noise Model}

The noise at all nodes consists of both background and impulsive noise components which are modeled using the Bernoulli-Gaussian noise model [15]. In this model, the background component, $n_{w}$, is considered complex Gaussian with zero mean and variance $\sigma_{w}^{2}$, whereas the impulsive part, $n_{i}$, is modeled as a Bernoulli-Gaussian random process. Hence,

$$
n=n_{w}+n_{i}
$$

while $n$ is the total noise component, $n_{i}=\mathrm{b} g$ where $g$ is complex white Gaussian noise with mean zero and $b$ is the Bernoulli process with probability mass function

$$
\operatorname{Pr}(\mathrm{b})= \begin{cases}p, & \mathrm{~b}=1 \\ 1-p, & \mathrm{~b}=0\end{cases}
$$

and $p$ is the probability occurrence of impulsive noise. Therefore, the PDF of the total noise can be simply expressed as

$$
P_{n}(n)=(1-p) \mathcal{C N}\left(n, 0, \sigma_{w}^{2}\right)+p \mathcal{C N}\left(n, 0, \sigma_{w}^{2}+\sigma_{i}^{2}\right)
$$

where $\mathcal{C N}$ is the Gaussian PDF. The variances $\sigma_{w}^{2}$ and $\sigma_{i}^{2}$ basically define the input signal-to-background noise ratio (SBNR) and the signal-to-impulsive noise ratio (SINR), respectively, as follows SBNR $=10 \log _{10}\left(1 / \sigma_{w}^{2}\right)$ and SINR $=$ $10 \log _{10}\left(1 / \sigma_{i}^{2}\right)$.

\section{Relaying and EH Protocol}

In our system model, it is assumed that the direct sourceto-destination link is strongly attenuated and hence all communications are accomplished over two phases via the relay. As mentioned in the introduction, although there are many 


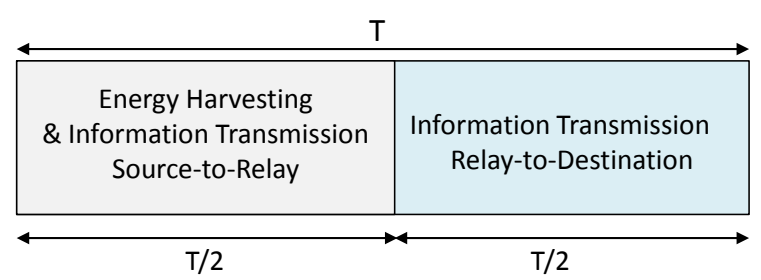

Figure 2: Time frame structure in the ideal relaying protocol.

EH protocols presented in the open literature, in this study, for simplicity, we will consider the ideal relaying receiver. This relaying protocol is capable of, concurrently, processing information and harvesting energy as can be deducted from the time frame structure shown in Fig. 2. To elaborate, in the first half of the time block, $T / 2$, the relay both processes the source information and harvests energy which is then used along with the relay's external power supply, $P_{r e}$, to amplify and forward the source signal to the destination node in the second $T / 2$ period. More details will be provided in the next section $^{3}$.

\section{Ergodic Spectral Efficiency}

To simplify our analysis of ergodic spectral efficiency, $\xi$, over the impulsive noise PLC channel, we consider the upper bound at the destination with the assumption that perfect knowledge of $p$ is available at the destination. In this case, $\xi$ can be expressed as [19]

$$
\xi=\frac{1}{2}\left((1-p) \mathbb{E}\left[\log _{2}\left(1+\gamma_{d}\right)\right]+p \mathbb{E}\left[\log _{2}\left(1+\frac{\gamma_{d}}{\beta}\right)\right]\right)
$$

while $\beta=1+\sigma_{i}^{2} / \sigma_{w}^{2}$ and $\gamma_{d}$ denotes the SBNR at the destination. It is worth pointing out that the pre-log factor $\frac{1}{2}$ is due to the required two time slots for source-to-destination transmission. Using the definition in (8), the EE can be expressed as

$$
\eta=\frac{\xi}{P_{t}}
$$

where $P_{t}=P_{s}+P_{r}$ is the total power consumption, $P_{s}$ and $P_{r}$ are the source and relay transmit powers.

\section{ENERGY EFFICIENCY ANALYSIS}

In this section we derive an analytical expression for the EE. To begin with, the received signal at the relay in the first phase can be expressed as [20]

$$
y_{r}=\sqrt{P_{s}} \exp \left(-\alpha d_{1}\right) h_{1} s+n_{r},
$$

where $s$ is the information signal normalized as $\mathbb{E}\left[|s|^{2}\right]=1$ and $n_{r}$ is the noise at relay node with variance $\sigma_{r}^{2}$. Similarly, the harvested energy at the relay can be written as

\footnotetext{
${ }^{3}$ This paper neglects the power consumed by the circuitry to process data at the relay and therefore all the available relay power will be used to forward the source signal.
}

$$
E_{H}=\frac{\kappa T}{2}\left[P_{s} \exp \left(-\alpha d_{1}\right) h_{1}^{2}+\sigma_{r}^{2}\right]
$$

where $0<\kappa<1$ is the EH efficiency determined mainly by the circuitry. In the second phase, the transmitted signal at the relay after the base-band processing and amplification is given by

$$
r=\sqrt{P_{s}} \exp \left(-\alpha d_{1}\right) G h_{1} s+G n_{r}
$$

where $G$ is the relay gain. Now, the received signal at the destination can be expressed as

$$
\begin{aligned}
y_{d}=\underbrace{\sqrt{P_{s} P_{r}} \exp \left(-\alpha\left(d_{1}+d_{2}\right)\right) h_{1} h_{2} G s}_{\text {Signal Part }} \\
+\underbrace{\sqrt{P_{r}} \exp \left(-\alpha d_{1}\right) h_{2} G n_{r}+n_{d}}_{\text {Overall Noise }}
\end{aligned}
$$

where

$$
P_{r}=P_{r e}+P_{r h}
$$

and $P_{r e}$ is the relay power from the external power supply and $P_{r h}$ is the harvested energy which is calculated as

$$
P_{r h}=\frac{2 E_{H}}{T}=\kappa\left[P_{s} \exp \left(-2 \alpha d_{1}\right) h_{1}^{2}+\sigma_{r}^{2}\right]
$$

Now, grouping the information and noise terms in (13), we can obtain the SNR at the destination as

$$
\gamma_{d}=\frac{P_{s} P_{r} \exp \left(-2 \alpha\left(d_{1}+d_{2}\right)\right) G^{2} h_{1}^{2} h_{2}^{2}}{P_{r} \exp \left(-2 \alpha d_{1}\right) \sigma_{r}^{2} G^{2} h_{2}^{2}+\sigma_{d}^{2}} .
$$

To simplify our analysis here, we consider at this stage only the case when $P_{r h}=0$, i.e. $P_{r}=P_{r e}$. With this in mind, we can rewrite (15) as

$$
\gamma_{d}=\frac{c_{1} h_{1}^{2} h_{2}^{2}}{c_{3} h_{2}^{2}+c_{2}}
$$

where

$$
\begin{aligned}
& c_{1}=P_{s} P_{r} \exp \left(-2 \alpha\left(d_{1}+d_{2}\right)\right) G^{2}, \\
& c_{2}=\sigma_{d}^{2}, \\
& c_{3}=P_{r} \exp \left(-2 \alpha d_{1}\right) \sigma_{r d}^{2} G^{2}, \\
& \mathcal{X}=c_{1} X, \text { and } \\
& \mathcal{Y}=c_{2} \bar{Y},
\end{aligned}
$$

where $X=h_{1}^{2}$ and $\bar{Y}=h_{2}^{-2}$. Given these definitions, the SNR $\gamma_{d}$ in (16) can be re-expressed in a simpler form as follows

$$
\gamma_{d}=\frac{\mathcal{X}}{\mathcal{Y}+c_{3}}
$$

Hence, using (8) and (18), $\xi$ can be expressed as

$$
\begin{aligned}
\mathbb{E}[\xi]=\frac{1}{2}\left((1-p) \mathbb{E}\left[\log _{2}\left(1+\frac{\mathcal{X}}{\mathcal{Y}+c_{3}}\right)\right]\right. \\
\left.+p \mathbb{E}\left[\log _{2}\left(1+\frac{\mathcal{X}}{\left(\mathcal{Y}+c_{3}\right) \beta}\right)\right]\right)
\end{aligned}
$$




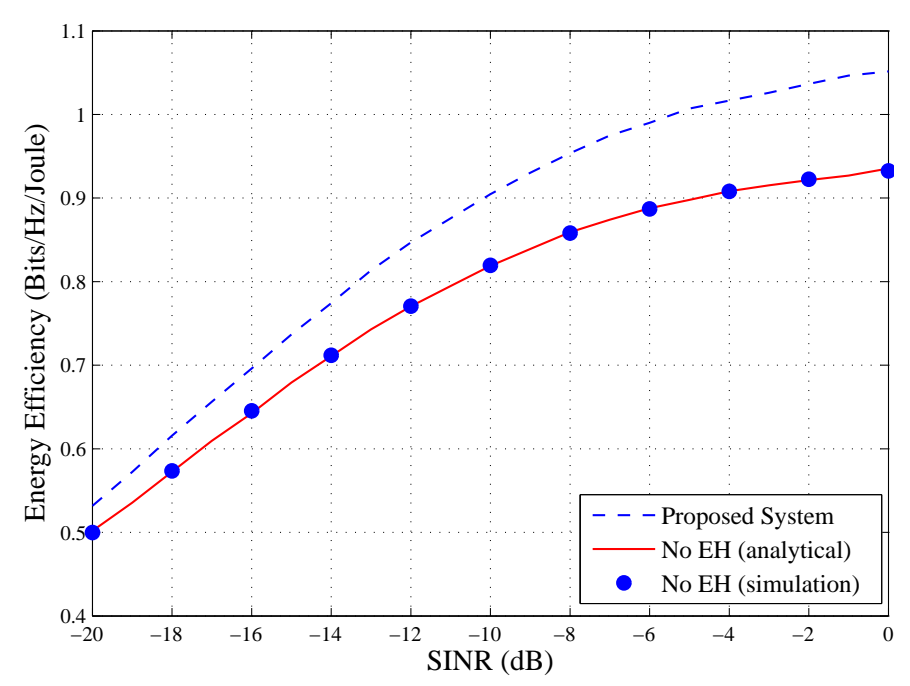

Figure 3: EE performance versus SINR for the proposed EH-based system and the conventional relaying approach.

Lemma 1. It is found in [21] that for any $u, v>0$

$$
\mathbb{E}\left[\ln \left(1+\frac{u}{v}\right)\right]=\int_{0}^{\infty} \frac{1}{s}\left(1-\Psi_{u}(s)\right) \Psi_{v}(s) d s,
$$

where $\Psi_{u}(s)$ and $\Psi_{v}(s)$ denote the MGFs of the random variables $u$ and $v$, respectively.

Using this definition, and since $\mathcal{X}$ and $\mathcal{Y}$ are independent, the destination spectral efficiency in (19) can be obtained as

$$
\begin{aligned}
\mathbb{E}[\xi]=\frac{1}{2 \ln (2)} & {\left[\int_{0}^{\infty} \frac{1-p}{s}\left(1-\Psi_{\mathcal{X}}(s)\right) \Psi_{\mathcal{Y}+c_{3}}(s) d s\right.} \\
& \left.+\int_{0}^{\infty} \frac{p}{s}\left(1-\Psi_{\mathcal{X}}(s)\right) \Psi_{\left(\mathcal{Y}+c_{3}\right) \beta}(s) d s\right]
\end{aligned}
$$

where $\Psi_{\mathcal{X}}(s), \Psi_{\mathcal{Y}+a_{3}}(s)$ and $\Psi_{\left(\mathcal{Y}+c_{3}\right) \beta}$ are the MGFs of the random variables $\mathcal{X}, \mathcal{Y}+a_{3}$ and $\left(\mathcal{Y}+a_{3}\right) \beta$, given, respectively, by [22]

$$
\begin{gathered}
\Psi_{\mathcal{X}}(s)=\Psi_{X}\left(c_{1} s\right) \\
\Psi_{\mathcal{Y}+c_{3}}(s)=\Psi_{\bar{Y}}\left(c_{2} s\right) \exp \left(-c_{3} s\right),
\end{gathered}
$$

and

$$
\Psi_{\left(\mathcal{Y}+c_{3}\right) \beta}(s)=\Psi_{\bar{Y}}\left(c_{2} \beta s\right) \exp \left(-c_{3} \beta s\right) .
$$

Since $X$ and $\bar{Y}$ are log-normally distributed with parameters $h_{1}^{2} \sim \mathcal{L} \mathcal{N}\left(2 \mu_{h_{1}}, 4 \sigma_{h_{1}}^{2}\right)$ and $h_{2}^{-2} \sim \mathcal{L} \mathcal{N}\left(-2 \mu_{h_{2}}, 4 \sigma_{h_{2}}^{2}\right)$, using the series expansion based on Gauss-Hermite integration (4), we can express $\Psi_{\mathcal{X}}(s), \Psi_{\mathcal{Y}+c_{3}}(s)$ and $\Psi_{\left(\mathcal{Y}+c_{3}\right) \beta}$ as in (25), (26) and (27), respectively, shown at the top of the next page. Finally, by substituting (25)-(27) into (21) and then into (9), we get the EE of the system under consideration.

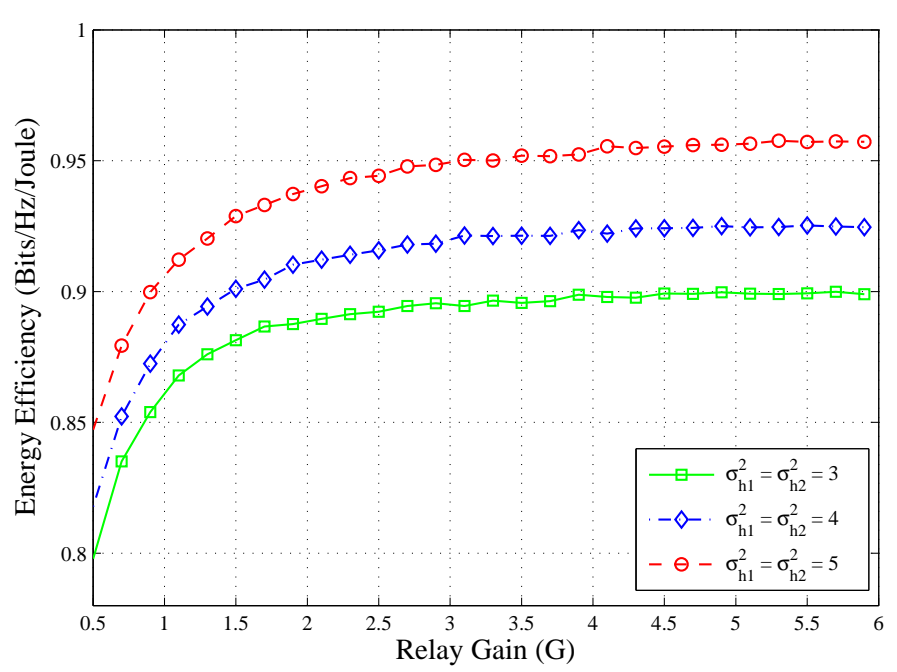

Figure 4: EE performance as a function of the relay gain for various channel coefficients for the proposed system when SINR $=-10 \mathrm{~dB}$.

\section{NUMERICAL RESUlTS}

This section presents some numerical and simulated examples of the proposed system above with different channel and noise scenarios. To validate the expression derived above, Monte Carlo simulations are provided. The system parameters considered here are as follows: $P_{s}=1$ watt, $P_{r e}=1$ watt, $G=1, \kappa=1, d_{1}=d_{2}=10 \mathrm{~m}, \mu_{h_{1}}=\mu_{h_{2}}=2 \mathrm{~dB}$, $\sigma_{h_{1}}^{2}=\sigma_{h_{2}}^{2}=5 \mathrm{~dB}, p=0.01$ and SBNR at all nodes is $10 \mathrm{~dB}$. In addition, the cable attenuation parameters used in these investigations are $a_{0}=9.4 \times 10^{-3}, a_{1}=4.2 \times 10^{-7}$ and $k=0.7$. It is worthwhile mentioning that these values represent an indoor PLC scenario as discussed in [1].

Fig. 3 depicts the EE of the proposed and the conventional relaying systems. The first observation one can clearly see is that the proposed system always has better EE performance in comparison to the conventional relaying-based system. It is also apparent that as SINR increases the performance of both systems under consideration deteriorates and the improvement gap becomes smaller.

Furthermore, to illustrate the impact of relay gain and channel coefficients on the performance of the proposed system, we plot in Fig. 4 the EE versus the relay gain for various channel gains from which one can see that increasing the relay gain will result in better performance for all the channel variances considered. It is also noticeable that when the relay gain is relatively high, the EE levels off irrespective of the channel gain value. The other remark on these results is that as both the source-to-relay and relay-to-source channel variances are increased, the system EE enhances.

\section{CONClusion}

In this paper we analyzed the EE performance of a dualhop cooperative relaying PLC system. To improve the EE of such systems, we proposed AF relaying with EH. For the sake of simplicity, we assumed ideal EH at the relay. An accurate analytical expression for the EE was derived and validated with Monte Carlo simulations. Results have shown that considerable improvements can be attained with 


$$
\Psi_{\mathcal{X}}(s) \triangleq \sum_{n=1}^{N} \frac{w_{n}}{\sqrt{\pi}} \exp [\underbrace{-P_{s} P_{r} \exp \left(-2 \alpha\left(d_{1}+d_{2}\right)\right) G^{2}}_{c_{1}} s \exp \left(\frac{\sqrt{2} 2 \sigma_{h_{1}} x_{n}+2 \mu_{h_{1}}}{\zeta}\right)]
$$

$$
\Psi_{\mathcal{Y}+c_{3}}(s) \triangleq \exp (\underbrace{-\exp \left(-2 \alpha\left(d_{1}+d_{2}\right)\right) P_{r} \sigma_{r}^{2} G^{2}}_{c_{3}} s) \sum_{n=1}^{N} \frac{w_{n}}{\sqrt{\pi}} \exp [\underbrace{-\sigma_{d}^{2}}_{c_{2}} s \exp \left(-\frac{\sqrt{2} 2 \sigma_{h_{2}} x_{n}+2 \mu_{h_{2}}}{\zeta}\right)]
$$

$$
\Psi_{\left(\mathcal{Y}+c_{3}\right) \beta}(s) \triangleq \exp (\underbrace{-\exp \left(-2 \alpha d_{2}\right) P_{r} \sigma_{r}^{2} G^{2} \beta}_{c_{3}} s) \sum_{n=1}^{N} \frac{w_{n}}{\sqrt{\pi}} \exp [\underbrace{-\beta \sigma_{d}^{2}}_{c_{2}} s \exp \left(-\frac{\sqrt{2} 2 \sigma_{h_{2}} x_{n}+2 \mu_{h_{2}}}{\zeta}\right)]
$$

the proposed system and this enhancement becomes more significant in moderate impulsive noise environments.

\section{ACKNOWLEDGMENT}

This work was funded by Innovate UK as part of the "Smart In-building Micro Grid for Energy Management" (Innovate UK project 101836).

\section{REFERENCES}

[1] M. Zimmermann and K. Dostert, "A multipath model for the powerline channel," IEEE Trans. Commun., vol. 50, pp. 553-559, Apr. 2002.

[2] A. Tonello, F. Versolatto, B. Bejar, and S. Zazo, "A fitting algorithm for random modeling the PLC channel," IEEE Trans. Power Del., vol. 27, pp. 1477-1484, Jul. 2012.

[3] K. M. Rabie and E. Alsusa, "Preprocessing-based impulsive noise reduction for power-line communications," IEEE Trans. Power Del., vol. 29, pp. 1648-1658, Aug. 2014.

[4] K. M. Rabie and E. Alsusa, "Quantized peak-based impulsive noise blanking in power-line communications," IEEE Trans. Power Del., vol. 29, pp. 1630-1638, Aug. 2014.

[5] N. A. T. O. Research and T. Organisation, "HF interference, procedures and tools - final report of NATO RTO information systems technology, panel research task group IST - 050/RTG-022,' Jun. 2007. 12, 34, 39, $41,128$.

[6] L. Lampe and A. Vinck, "Cooperative multihop power line communications," in Proc. IEEE Int. Symp. Power Line Commun. and Its Appl. (ISPLC), pp. 1-6, Mar. 2012.

[7] A. Tonello, F. Versolatto, and S. D'Alessandro, "Opportunistic relaying in in-home PLC networks," in Proc. IEEE Global Telecommun. Conf. (GLOBECOM), pp. 1-5, Dec. 2010.

[8] X. Cheng, R. Cao, and L. Yang, "Relay-aided amplify-and-forward powerline communications," IEEE Trans. Smart Grid, vol. 4, pp. 265 272, Mar. 2013.

[9] W. Bakkali, M. Tlich, P. Pagani, and T. Chonavel, "A measurementbased model of energy consumption for PLC modems," in Proc. IEEE Int. Symp. Power Line Commun. and Its Appl. (ISPLC), pp. 42-46, Mar. 2014.

[10] H. Gacanin, B. Adebisi, and A. Ligata, "Energy-efficient multipledomain bidirectional scheme for G.hn applications," Int. J. of Commun. Syst., 2015.
[11] H. Sun, A. Nallanathan, N. Zhao, and C.-X. Wang, "Green data transmission in power line communications," in Proc. IEEE Global Commun. Conf. (GLOBECOM), pp. 3702-3706, Dec. 2012.

[12] S. D'Alessandro and A. M. Tonello, "On rate improvements and power saving with opportunistic relaying in home power line networks," EURASIP J. on Advances in Signal Process., vol. 2012, pp. 1-17, Sept. 2012.

[13] S. D'Alessandro, A. Tonello, and F. Versolatto, "Power savings with opportunistic decode and forward over in-home PLC networks," in Proc. IEEE Int. Symp. Power Line Commun. and Its Appl. (ISPLC), pp. 176181, Apr. 2011.

[14] W. Bakkali, P. Pagani, and T. Chonavel, "Energy efficiency performance of relay-assisted power-line communication networks," in Proc. IEEE Consumer Commun. and Networking Conf. (CCNC), pp. 525-530, Jan. 2015.

[15] M. Ghosh, "Analysis of the effect of impulse noise on multicarrier and single carrier QAM systems," IEEE Trans. Commun., vol. 44, pp. 145147, Feb. 1996.

[16] M. K. Simon and M.-S. Alouini, Digital Communication Over Fading Channels. 2nd ed. Hoboken, NJ, USA: Wiley, 2005.

[17] N. Mehta, J. Wu, A. Molisch, and J. Zhang, "Approximating a sum of random variables with a lognormal," IEEE Trans. Wireless Commun., vol. 6 , pp. 2690-2699, Jul. 2007.

[18] M. Abramowitz and I. A. Stegun, Handbook of Mathematical Functions with Formulas, Graphs, and Mathematical Tables. ser. App. Math. Series 55. Washington, DC, USA: Nat. Bur. Stand., 1972.

[19] H. Vu, N. Tran, T. Nguyen, and S. Hariharan, "Estimating shannon and constrained capacities of Bernoulli-Gaussian impulsive noise channels in Rayleigh fading," IEEE Trans. Commun., vol. 62, pp. 1845-1856, Jun. 2014.

[20] H.-M. Wang, M. Luo, X.-G. Xia, and Q. Yin, "Joint cooperative beamforming and jamming to secure AF relay systems with individual power constraint and no eavesdropper's CSI," IEEE Signal Process. Lett., vol. 20, pp. 39-42, Jan. 2013.

[21] K. Hamdi, "A useful lemma for capacity analysis of fading interference channels," IEEE Trans. Commun., vol. 58, pp. 411-416, Feb. 2010.

[22] S. M. ROSS, Introduction to probability models. 10th ed. San Diego, CA: Academic, 2010 